\title{
Análise do comportamento de autocuidado de homens diagnosticados com Diabetes Mellitus Tipo II
}

\section{Analysis of the behavior of self-care for men diagnosed with Type II Diabetes Mellitus}

\author{
Míria Rita Duarte \\ Faculdades Integradas Pitágoras de Montes Claros - FIPMoc - Montes Claros - Brasil \\ miriaritaduarte@gmail.com \\ Julliana Andrade do Carmo \\ Faculdades Integradas Pitágoras de Montes Claros - FIPMoc - Montes Claros - Brasil \\ jullianacarmo@hotmail.com \\ Valker Santiago Goes Filho \\ Faculdades Integradas Pitágoras de Montes Claros - FIPMoc - Montes Claros - Brasil \\ valker_santiago@hotmail.com \\ Maria Luiza Terra Santos \\ Faculdades Integradas Pitágoras de Montes Claros - FIPMoc - Montes Claros - Brasil \\ luiza-maria@hotmail.com \\ Janimar Lago \\ Faculdades Integradas Pitágoras de Montes Claros - FIPMoc - Montes Claros - Brasil \\ janimar.lago@hotmail.com \\ Ronilson Ferreira Freitas \\ Faculdades Integradas Pitágoras de Montes Claros - FIPMoc - Montes Claros - Brasil \\ ronnypharma@bol.com.br \\ Vivianne Margareth Chaves Pereira Reis \\ Faculdades Unidas do Norte de Minas - FUNORTE - Montes Claros - Brasil \\ viola.chaves@yahoo.com.br \\ Daniela Araújo Veloso Popovic \\ Universidade Estadual de Montes Claros- Unimontes - Montes Claros - Brasil \\ Faculdades Unidas do Norte de Minas - FUNORTE - Montes Claros - Brasil \\ danielaveolloso@yahoo.com.br \\ Josiane Santos Brant Rocha \\ Faculdades Integradas Pitágoras de Montes Claros - FIPMoc - Montes Claros - Brasil \\ Universidade Estadual de Montes Claros- Unimontes - Montes Claros - Brasil \\ josianenat@yahoo.com.br
}

\section{RESUMO}

OBJETIVO: Verificar o comportamento de autocuidado em homens com Diabetes Mellitus tipo II, na cidade de Montes Claros/MG. 
MÉTODOS: O presente estudo caracteriza-se por ser descritivo, do tipo corte transversal e de caráter qualitativo. A amostra foi constituída por 39 indivíduos portadores de Diabetes Mellitus tipo II, do sexo masculino, com faixa etária compreendida entre 40 e 60 anos. Trata-se de um estudo que abrangeu a avaliação sociodemográfica, antropométrica, da glicemia e do autocuidado. Como estratégia de coleta de dados foram aplicados questionários e realizados testes glicêmicos, que acompanharam a obtenção de medidas de peso, altura, circunferência da cintura e quadril.

RESULTADOS: Os resultados obtidos na avaliação clínica, o índice de massa corporal, a relação cintura-quadril, bem como a glicemia, apresentaram médias que estão acima do preconizado em um indivíduo saudável.

CONCLUSÕES: O comportamento de autocuidado dos homens diagnosticados com Diabetes Mellitus Tipo II se encontra indesejável para atitudes indispensáveis no cuidado a esse tipo de doença, como a monitoração da glicemia e a realização de atividade física. Entretanto, quanto à alimentação e ao uso de medicamentos os resultados se mostram favoráveis.

PALAVRAS-CHAVE: Comportamento. Autocuidado. Diabetes Mellitus.

\section{ABSTRACT}

OBJECTIVE: Check the behavior of self-care in men with type II Diabetes Mellitus in the city of Montes Claros/MG.

METHODS: This study is characterized by being descriptive and cross-sectional and qualitative nature. The sample was composed of individuals with Diabetes Mellitus type II, male, aged between 40 and 60 years. It is a study that covered the assessment socio-demographic, anthropometric assessment, evaluation of blood glucose levels and evaluation of self-care. As a strategy for collecting questionnaires were applied and performed blood glucose test, which accompanied the obtaining measures of weight, height, circumference of the hips and waist.

RESULTS: The results obtained in the clinical evaluation, BMI, WHR, as well as the glucose, averages that are above the recommended in a healthy individual.

CONCLUSIONS: The behavior of self-care of men diagnosed with type II Diabetes Mellitus is undesirable for indispensable attitudes in care to this type of disease such as monitoring blood glucose levels and the performance of physical activity, however, as the supply and the use of medicines the results are favorable.

KEYWORDS: Behavior. Self-care. Diabetes Mellitus.

\section{Introdução}

A Diabetes Mellitus constitui uma desordem metabólica de etiologia múltipla caracterizada por hiperglicemia crônica proveniente de alterações na secreção ou ação da insulina, resultando em redução da captação de glicose pelos tecidos periféricos (FERREIRA et al., 2011). Carvalho et al. (2012) acrescentam que a presença de hiperglicemia, frequentemente acompanhada de dislipidemia, obesidade abdominal, hipertensão arterial e disfunção endotelial, também compõem a caracterização dessa patologia. A associação dessas manifestações são fatores desencadeadores de outras complicações, como doenças cardiovasculares e microangiopatias com comprometimento da retina, glomérulo e nervos (FERREIRA et al., 2011).

A Diabetes Mellitus possui uma alta prevalência, além de ser responsável por perdas importantes da qualidade de vida e representar um gasto monetário muito grande para o sistema de saúde, uma vez que indivíduos diabéticos apresentam cerca de 2,4 vezes mais despesas médicas que indivíduos não diabéticos da mesma idade, raça e sexo (PANAROTTO; TELES; SCHUMACHER, 2008). 
Segundo Carvalho et al. (2012), o aumento da prevalência da diabetes está relacionado ao envelhecimento da população, à obesidade crescente, mudanças alimentares e no estilo de vida marcados pelo aumento da ingestão de gorduras, açúcares e sedentarismo. A dieta e o exercício são fundamentais para o tratamento dessa doença, porém devem ser empregadas em um plano individualizado que exige a adesão do paciente. As mudanças alimentares envolvem ingestão de alimentos com baixo índice glicêmico e ricos em fibras alimentares, além dos exercícios que corroboram para redução da hemoglobina glicada, aumento da sensibilidade dos tecidos à insulina e redução dos níveis circulantes de triglicérides e LDL. Ocorre associação de terapia medicamentosa quando as mudanças de hábitos não são suficientes para manutenção do controle (CODOGNO; FERNANDES; MONTEIRO, 2012). Devido à característica crônica da doença, os diabéticos apresentam riscos de desenvolverem alguma complicação que aliada ao envelhecimento interferem na adesão ao autocuidado e torna a terapia mais complexa (GOMES-VILLAS BOAS et al., 2011).

Optou-se por essa população visto que, como salientado por Modeneze et al. (2012), o estilo de vida contemporâneo tem gerado problemas de saúde na população em geral, com crescente ocorrência de doenças crônicas não transmissíveis. Sendo destacado por Lojudice et al. (2008 apud MODENEZE et al., 2012) a tendência elevada da Diabetes Mellitus Tipo II, com projeções no Brasil fornecidas pelo Ministério da Saúde, da existência de aproximadamente cinco milhões de portadores de Diabetes Mellitus, sendo aproximadamente $90 \%$ destes portadores do Tipo II e $10 \%$ do Tipo I.

Como agravo dessa situação, o homem como provedor familiar alega dificuldade de participar dos programas de prevenção e recuperação oferecido pela atenção primária, já que as consultas e eventos coincidem com o horário de trabalho, segundo informa o Ministério da Saúde (2008).

Diante da dificuldade do homem de se cuidar, esse estudo objetiva verificar o comportamento de autocuidados em homens com Diabetes Mellitus tipo II na cidade de Montes Claros/MG.

\section{Metodologia}

O presente estudo caracteriza-se por ser descritivo, do tipo corte transversal, de cunho quantitativo. Os critérios de inclusão compreenderam pacientes do sexo masculino, com idade entre 40 e 65 anos, diagnosticados com Diabetes Mellitus Tipo II, assistidos pelo programa de assistência ao diabético ofertado pelas Estratégias de Saúde da Família, conveniadas às Faculdades Integradas Pitágoras de Montes Claros - FIPMoc, no ano de 2012. A amostra foi constituída por 39 indivíduos portadores de Diabetes Mellitus Tipo II, do sexo masculino, com faixa etária compreendida entre 40 e 60 anos.

A coleta dos dados foi realizada no período de outubro a novembro de 2012, em Montes Claros, uma cidade de médio porte, localizada na região Norte do Estado de Minas Gerais.

A pesquisa abrangeu a avaliação sociodemográfica, antropométrica, da glicemia e do autocuidado.

Dentre os dados coletados na avaliação sociodemográfica estavam idade, raça, escolaridade, renda familiar, estado civil, participação em grupos de diabéticos e prática de atividade física.

Como estratégia de coleta de dados foram aplicados questionários e realizados testes glicêmicos, que acompanharam a obtenção de medidas de peso, altura, circunferência do quadril e cintura.

Para avaliação antropométrica foi utilizado o cálculo do Índice de Massa Corporal (IMC) $\left(\mathrm{IMC}=\right.$ peso/altura $\left.{ }^{2}\right)$, utilizando os dados de medida de peso e altura coletados durante a entrevista. Para análise IMC considerou-se a classificação da WHO (2010): não obeso (27,8 a 31,1 Kg/m²) e obeso $\left(\geq 31,1 \mathrm{Kg} / \mathrm{m}^{2}\right)$. Ainda foram coletadas as medidas de circunferência de cintura e quadril, considerando como ponto de corte a relação cintura-quadril (RCQ) de 0,95 (WHO, 2010).

Foi realizado, também, um teste glicêmico em jejum, seguido por uma avaliação de autocuidade, sendo que esta última compreendeu um questionário validado de Avaliação de 
Autocuidado com Diabetes, versão traduzida para Portugal por Bastos, Severo e Lopes (2007), da escala Summary of diabetes self-careactivitiesmeasures (SDSCA), de Glasgow, Toobert e Hampson (2000).

Para caracterizar o universo amostral pesquisado foi utilizada análise descritiva com dados de média e desvio padrão além de porcentagens. Para encontrar a associação entre as variáveis foi utilizado o teste Chi-Square. Foi considerado como nível de significância $\mathrm{p}<0,05$, estabelecido pelo pacote Statisticalpackage for the social science (SPSS), versão 14.0.

O Projeto de Pesquisa foi submetido para apreciação do Comitê de Ética em Pesquisa com seres humanos, das Faculdades Integradas Pitágoras de Montes Claros, e aprovado sob parecer $038757 / 2012$.

\section{Resultados}

A distribuição dos participantes com relação às variáveis sociodemográficas (raça, instrução, estado civil, uso de tabaco e renda familiar) encontra-se na Tabela 1:

Tabela 1 - Analise descritiva sóciodemográfica da amostra analisadaFatores

\begin{tabular}{lll}
\hline \multicolumn{1}{c}{ Sociodemográficos } & \multicolumn{1}{c}{ Variáveis } & $(\%)(\mathbf{n})$ \\
\hline Raça & Branca & $53,8-21$ \\
& Parda & $33,3-13$ \\
& Negra & $12,9-5$ \\
Instrução & Menos de 7 anos de estudo formal & $71,8-28$ \\
& Mais de 7 anos de estudo formal & $28,2-11$ \\
Tabaco & Fumante & $12,8-5$ \\
& Não Fumante & $87,2-34$ \\
Estado Civil & Solteiro & $17,9-7$ \\
& Casado & $76,9-30$ \\
& Viúvo & $5,2-2$ \\
& & \\
Renda Familiar & Menos de 2 salários & $69,9-27$ \\
& De 2 a 5 salários & $25,6-10$ \\
& Mais de 5 salários & $4,5-2$ \\
\hline
\end{tabular}

Nota: (\%) - Porcentagem observada; (n) - Tamanho da Amostra.

Fonte: Autoria própria (2012).

Tabela 2:

Os dados referentes a antropometria (idade, peso, IMC, RCQ e glicemia) estão dispostos na

Tabela 2 - Média e desvio padrão das variáveis

antropométricas e glicemia da amostra analisada

\begin{tabular}{cl}
\hline $\begin{array}{c}\text { Fatores antropométricos - } \\
\text { Clínicos }\end{array}$ & Média $( \pm \mathbf{D p})$ \\
\hline Idade & $61,56(8,82)$ \\
Peso & $86,19(18,81)$ \\
IMC & $29,91(5,03)$ \\
RCQ & $0,99(0,05)$ \\
Glicemia & $173,36(62,40)$ \\
\hline
\end{tabular}

Nota: Média; Dp - desvio-padrão.

Fonte: Autoria própria (2012).

Na Tabela 3 destacou-se a caracterização do comportamento de autocuidado da amostra com a média e o desvio padrão das variáveis: 
Tabela 3 - Média e desvio padrão do comportamento de autocuidado dos homens diabéticos tipo II

\begin{tabular}{clc}
\hline & Comportamento de autocuidado & Média (士 Dp) \\
\hline Alimentação Geral & Dieta Saudável & $4,85(2,56)$ \\
& Orientação do médico para alimentação & $4,26(2,89)$ \\
Alimentação Específica & Comeu 5 ou mais porções de frutas e vegetais & $4,85(1,96)$ \\
& Comeu gorduras, carnes vermelhas, leite integral & $4,26(2,75)$ \\
& Comeu doces & $1,54(2,30)$ \\
Atividade Física & Realizou atividade física contínua por pelo menos 30 minutos & $3,96(2,83)$ \\
& Praticou exercício físico específico (Exemplo: nadar, caminhar, correr. & $2,64(2,72)$ \\
& Excluindo atividades físicas de casa e trabalho) & $1,79(2,27)$ \\
Monitoração da GlicemiaAvaliou açúcar do sangue & $2,10(2,0)$ \\
& Avaliou açúcar do sangue o número de vezes que o médico recomendou & $5,95(1,98)$ \\
\hline
\end{tabular}

Notas: Média; Dp - desvio-padrão.

Fonte: Autoria própria (2012).

\section{Discussão}

De acordo com os dados caracterizados na Tabela 1, o item raça teve predominância da população branca $(53,8 \%)$. Tal predomínio contrasta com os dados obtidos por Coblentz et al. (2010), em seus estudos com pacientes diagnosticados com diabetes, assistidos no ambulatório de oftalmologia do Hospital Universitário Gaffrée e Guinle da Universidade Federal Fluminense do Rio de Janeiro. No referido estudo, os autores obtiveram uma população branca de apenas $36 \%$. Porém, destaca-se que não existe uma rígida separação entre os indivíduos por cor, pois esse conceito tem elevada interferência no Brasil, que se caracteriza por apresentar elevada taxa de miscigenação e percepção de cor influenciada por aparência física, prestígio social e tipo regional de sociedade (BRASIL, 2008).

O grau de instrução da população investigada mostrou que $71,8 \%$ dos entrevistados tinham menor que sete anos de estudo formal, o que vai de encontro com as observações de Lima, Pereira e Romano (2011), que descrevem uma média de grau de escolaridade para os indivíduos diabéticos, do sexo masculino de 6,4 \pm 4,6 anos de estudos. Ochoa-Vigo et al. (2006) destacam que a baixa instrução facilita o desencadeamento de doenças crônicas, devido à dificuldade de acesso as informações e de compreensão das medidas preventivas. A afirmação pauta-se nos resultados obtidos em estudo sobre o perfil dos diabéticos acometidos por ulcerações nos pés, em que $87 \%$ da amostra possuía ensino fundamental incompleto, tendo esse dado um caráter fundamental para os autores em questão.

Os homens entrevistados afirmaram, em sua maioria, estarem casados (76,9\%). Este resultado vai de encontro com os obtidos por Lima, Pereira e Romano (2011), que constataram em trabalho realizado com 154 diabéticos, o predomínio do estado civil casado entre estes $(72,9 \%)$. O estado civil interfere na adesão ao tratamento, na dinâmica familiar e o perfil psicossocial influencia nas variações glicêmicas, sendo o ambiente familiar um estímulo ao autocuidado, influenciando no comportamento no que diz respeito ao enfrentamento da doença e controle metabólico (OTERO; ZANETTI; TEIXEIRA, 2007).

Quanto à idade dos indivíduos analisados, a média de 61,56 $( \pm 8,82)$ está pertinente com a literatura pois esta destaca que a frequência da doença aumenta gradativamente após os 50 anos (GRILLO; GORONI, 2007; OTERO; ZANETTI; TEIXEIRA, 2007), dado que confirma a afirmativa de Freitas (2011), que em sua pesquisa concluiu que no Brasil a prevalência da diabetes é acentuada na faixa etária de 60 aos 79 anos. 
Devido à natureza crônica do diabetes mellitus, os portadores apresentam risco de desenvolvimento de complicações tardias que, associadas ao envelhecimento, podem afetar a adesão ao autocuidado e ao tratamento (GOMES-VILAS BOAS et al., 2011).

Os entrevistados obtiveram média de peso corporal $86,19 \mathrm{Kg}( \pm 18,81)$, resultado que mostra-se de acordo com o obtido por Torres, Pace e Stradioto (2010), em seu estudo no seguimento ambulatorial do hospital de referência de Belo Horizonte, onde os pacientes apresentaram média de peso de $74,52 \mathrm{Kg}( \pm 16,60)$.

$\mathrm{O}$ dado relativo ao peso corporal frisa um aparente excesso de peso, com possível sobrepeso da população alvo da pesquisa, hipótese confirmada pelo valor de IMC onde se obteve a média de $29,91$ ( $\pm 5,03)$. Confirmando os valores alcançados por Torres, Pace e Stradioto (2010), cujos índices descritos de IMC 28,91 $( \pm 7,22)$ reforçam a identificação de um inadequado controle da diabetes.

O RCQ 0,99 cm ( $\mathrm{p}<0,05)$ mostra-se inadequado, visto que, segundo Guedes e Guedes (1997), é considerado normal o RCQ $\leq 0,85 \mathrm{~cm}$ para homens. Também reforça os achados deste estudo os resultados de Fiani, Monteiro e Foss (2009), que em estudo para avaliação da RCQ com glicemia e massa corpórea em diabéticos tipo II, observou que a RCQ contribui para regulação da glicemia. Os autores afirmam que o alto teor lipídico e acúmulo de gordura na região abdominal acompanham o aumento na glicemia e o risco de desenvolver diabetes mellitus, já que desencadeiam desordens metabólicas e resistência a insulina. De acordo com Fiani, Monteiro e Foss (2009), há uma correlação entre as variáveis RCQ e Glicemia, ocorrendo para cada aumento de 0,1 da RCQ uma elevação de $15 \mathrm{mg} / \mathrm{dL}$ na glicemia.

A glicemia da amostra apresentou uma média 173,36 $( \pm 62,4)$, o que não representa um valor coerente com um bom manejo da diabetes e tem intima relação com a monitorização de glicemia que obteve resultados indesejáveis, tanto para a avaliação de açúcar no sangue $(1,79)$, quanto para a avaliação da glicemia de acordo com a recomendação médica $(2,10)$. Tais resultados se encontram de acordo com os obtidos por Oliveira et al. (2012), que salientam em seu estudo, com amostra abrangendo 50 diabéticos tipo II, os valores de glicemia capilar no momento da inclusão no estudo, com variação de 212 a $345 \mathrm{mg} / \mathrm{dL}$.

Uma vez realizado o controle glicêmico, comprovadamente, pode-se reduzir os riscos de complicação micro e macro vasculares, o que é confirmado pelo estudo no qual o tratamento intensivo por meio da prática de atividades físicas, monitorização da glicemia e do percentual lipídico associados à dieta e cessação do tabagismo, obteve redução das taxas de doença cardiovascular, nefropatia, retinopatia e neuropatia entre os estudados (SOCIEDADE BRASILEIRA DE ENDOCRINOLOGIA E METABOLOGIA ASSOCIAÇÃO BRASILEIRA DE NUTROLOGIA, 2011).

Os resultados apresentados neste trabalho chamam a atenção para o inadequado controle metabólico dos pacientes diabéticos analisados, salientando uma má adesão ao tratamento desta patologia.

Quanto à alimentação geral, resultado favorável foi evidenciado, assim como no estudo similar relatado por Assunção e Ursine (2008), realizado em Belo Horizonte, onde 52,44\% dos entrevistados tinham adesão total ao tratamento não farmacológico, que envolveu o controle alimentar e prática de exercício físico, 38,41\% adesão parcial e 9,15\% sem adesão (ASSUNÇÃO; URSINE, 2008). Contudo, segundo pesquisa realizada por Assunção, Santos e Costa (2002), em 32 postos da rede de atenção primária à saúde, da zona urbana de Pelotas, constatou-se que aproximadamente $76 \%$ das pessoas entrevistadas receberam orientações alimentares, porém, apenas a metade seguiu as recomendações e realizou dieta nos últimos 15 dias, demonstrando uma adesão aproximada de $50 \%$, situação ainda inadequada ao autocuidado.

Com relação à alimentação específica, no que se refere à ingestão de frutas e vegetais, a amostra obteve resultados desejáveis $(4,26)$. Esse valor contrasta com os obtidos em pesquisa realizada com 67 diabéticos, que evidenciou ingestão de vegetais, frutas e laticínios insatisfatóris, considerando as recomendações. Os resultados salientaram o elevado número de pacientes que não atenderam às recomendações a respeito da dieta $(59,7 \%)$, ingerindo poucas porções de cereais, 
frutas, vegetais e laticínios, incluindo uma parcela considerável que apresentou um consumo excessivo de gorduras totais e saturadas, de colesterol e de sódio (SANTOS et al., 2009).

A ingestão de doces mostrou-se desejável $(1,54)$. De acordo com dados coletados na cidade de Florianópolis, em pesquisa realizada com 140 indivíduos, divididos em dois grupos (diabéticos e não diabéticos), pacientes diabéticos apresentam maior critério na escolha dos alimentos, evitando os alimentos que contribuem para o ganho de peso como pizza, lasanha e macarrão. $\mathrm{O}$ estudo relatou que a ingestão de alimentos doces por diabéticos, como doce de leite e sorvete, representou $2,9 \%$ e $20 \%$, respectivamente, enquanto os não diabéticos $28,6 \%$ e $41,4 \%$, salientando que a condição de saúde interfere na escolha alimentar e a orientação nutricional. Nesse sentido, auxilia na escolha adequada dos alimentos (IOP; TEIXEIRA; DELIZA, 2009).

A prática de atividade física mostrou uma situação não desejável entre a amostra investigada, 3,96 $( \pm 2,83)$ 2,64 $( \pm 2,72)$, respectivamente. Tal situação também é relatada por Miranzi et al. (2008) que em seus estudos com diabéticos, identificou que 27,8\% não praticavam exercícios físicos.

Quanto ao uso da medicação, a amostra apresenta resultados desejáveis para o uso correto de medicamentos (5,95). De acordo com Santos, Oliveira e Colete (2010), a média de medicamentos utilizados para tratamento de Diabetes Mellitus Tipo II, entre homens é de 2,7 $( \pm 0,8)$, valor superior ao encontrado entre as mulheres, $2,2( \pm 1)$.

Como destacam Faria et al. (2009) em estudo realizado com Diabéticos, em um Centro de Pesquisa e Extensão Universitária do interior paulista, constataram valores adequados de utilização do medicamento quanto à dose, horário, número de comprimidos e número de vezes da ingestão. Porém, verificaram que 56,5\% dos participantes apresentaram déficit no conhecimento acerca do medicamento para o controle do diabetes, fato preocupante que pode levar a agravos do estado de saúde desses.

Os resultados apresentados nesse estudo deixam claro que se faz necessário a conscientização do paciente e adesão do mesmo ao autocuidado para um bom manejo da Diabetes Mellitus Tipo II, o que, na população masculina, com a faixa etária pesquisada, não vem ocorrendo satisfatoriamente, exigindo identificação desse quadro por parte dos profissionais de saúde para reestruturação dos programas de educação e intervenção que abrangem tal população que se mostra de difícil acesso.

\section{Conclusão}

Com base nos resultados deste estudo, verifica-se que o comportamento de autocuidado dos homens diagnosticados com Diabetes Mellitus Tipo II se encontra indesejável para atitudes indispensáveis no cuidado a esse tipo de doença como a monitoração da glicemia e a realização de atividade física. Porém, destacou-se que quanto à alimentação e ao uso de medicamentos os resultados se mostram favoráveis.

Os resultados obtidos na avaliação clínica, o IMC, o RCQ, bem como a glicemia, apresentaram médias que estão acima do preconizado em um indivíduo saudável. A interpretação desses dados associada ao comportamento inadequado quanto às variáveis anteriormente citadas, confirmam a necessidade de esforços para a conscientização da população portadora de Diabetes Mellitus Tipo II para a prática do autocuidado. Além disso, espera-se que os profissionais de saúde da atenção primária, possam estabelecer metas a serem traçadas quanto ao manejo dessa doença e desse grupo populaçional em particular.

\section{Referências}

ASSUNÇÃO, M. C. F.; SANTOS, I. da S. dos; COSTA, J. S. D da. Avaliação do processo de atenção médica: adequação do tratamento de pacientes com diabetes mellitus, Pelotas, Rio Grande do Sul, Brasil. Caderno de Saúde Publica, Rio de Janeiro, v. 18, n. 1, p. 205-211, jan./fev. 2002. 
ASSUNÇÃO, T. S.; URSINE, P. G. S. Estudo de fatores associados à adesão ao tratamento não farmacológico em portadores de diabetes mellitus assistidos pelo Programa Saúde da Família, Ventosa, Belo Horizonte. Ciência e Saúde Coletiva, Rio de Janeiro, v. 13, n. 2, p. 2189-2197, 2008.

BASTOS, F.; SEVERO, M.; LOPES, C. Psychometric analysis of diabetes self-care scale (translated and adapted to Portuguese). Acta Médica Portuguesa, v. 20, n. 1, p. 11-20, 2007.

BRASIL. SECRETARIA DE ATENÇÃO À SAÚDE. Política Nacional de Atenção Interal à Saúde do Homem: Princípios e Diretrizes, Brasília, 2008. Disponível em: <http://dtr2001.saude.gov.br/sas/PORTARIAS/Port2008/PT-09-CONS.pdf>. Acesso em: 13 dez. 2012.

CARVALHO, F. S. et al. Importância da orientação nutricional e do teor de fibras da dieta no controle glicêmico de pacientes diabéticos tipo 2 sob intervenção educacional intensiva. Arquivo Brasileiro Endocrinologia e Metabologia, São Paulo, v. 56, n. 2, p. 110-119, 2012.

COBLENTZ, J. et al. Prevalência de diabetes mellitus tipo 2 e outros fatores de risco associados em pacientes com glaucoma. Revista Brasileira de Oftalmologia, Rio de Janeiro, v. 69, n. 1, p. 33-35, 2010.

CODOGNO, J. S.; FERNANDES, R. A.; MONTEIRO, H. L. Prática de atividades físicas e custo do tratamento ambulatorial de diabéticos tipo 2 atendidos em unidade básica de saúde. Arquivo Brasileiro Endocrinologia e Metabologia, São Paulo, v. 56, n. 1, p. 6-11, 2012.

FARIA,T. G. et al. Conhecimento sobre terapêutica medicamentosa em diabetes: um desafio na atenção à saúde. Acta Paulista de Enfermagem, São Paulo, v. 22, n. 5, p. 612-617, 2009.

FERREIRA, L. T. et al. Diabetes Mellitus: hiperglicemia crônica e suas complicações. Arquivos Brasileiros de Ciências da Saúde, São Paulo, v. 36, n. 3, p. 182-188, 2011.

FIANI, C. R. V; MONTEIRO, L. Z.; FOSS, C. Avaliação da relação cintura/quadril com glicemia e massa corpora em Diabéticos Tipo 2. Revista EpeQ/Fafibe, Bebedouro, v. 1, n. 1, p. 29-35, 2009.

FREITAS, R. W. J. F. de. Fatores associados à não adesão aos agentes antidiabéticos orais em paciente com diabetes mellitus tipo 2. Revista de la ALAD: Asociación Latinoamericana de Diabetes, Bogotá, v. 1, n. 4, p. 170-183, out./dez. 2011.

GLASGOW, R. E.; TOOBERT, D. J.; HAMPSON, S. E. The summary of diabetes self-care activities measure: results from 7 studies and a revised scale. Diabetes Care, v. 23, n. 7, p. 943950, 2000.

GOMES-VILLAS BOAS, L. C. et al. Adesão à dieta e ao exercício físico das pessoas com Diabetes Mellitus. Texto \& Contexto Enfermagem, Florianópolis, v. 20, n. 2, p. 272-279, 2011.

GRILLO, M. de F. F.; GORINI, M. I. P. C. Caracterização das Pessoas com Diabetes Mellitus tipo 2. Revista Brasileira de Enfermagem, Brasília, v. 60, n. 1, p. 49-54, 2007.

GUEDES, D. P.; GUEDES, J. E. R. P. Crescimento, composição corporal e desempenho motor em crianças e adolescentes. São Paulo: CLR Balieiro, 1997.

IOP, S. C. F.; TEIXEIRA, E.; DELIZA, R. Comportamento alimentar de indivíduos diabéticos. 
Brazilian Journal Food Technology, Campinas, Edição Especial - II Simpósio de Segurança Alimentar, 2009.

LIMA, A. P. de; PEREIRA, D. A. G.; ROMANO; V. F. Perfil sócio-demográfico e de saúde de idosos diabéticos atendidos na atenção primária. Revista Brasileira de Ciências da Saúde, São Caetano do Sul, v. 15, n. 1, p. 39-46, 2011.

MINISTÉRIO DA SAÚDE. Manual de doenças mais importantes por razões étnicas, na população brasileira Afro-Descendente. Brasília: Secretaria de Políticas de Saúde, 2008.

MIRANZI, S. de S. C. et al. Qualidade de vida de indivíduos com diabetes mellitus e hipertensão acompanhados por uma equipe de daúde da família. Texto \& Contexto Enfermagem, Florianópolis, v. 17, n. 4, p. 672-679, out./dez., 2008.

MODENEZE, D. M. et al. Nível de atividade física de portadores de diabetes mellitus tipo 2 (DM2) em comunidade carente no Brasil. Revista Medicina, Ribeirão Preto, v. 45, n. 1, p. 78-86, 2012. Disponível em: http://www.fmrp.usp.br/revista./. Acesso em: 25 out. 2012.

OCHOA-VIGO, K. Et al. Caracterização de pessoas com diabetes em unidades de atenção primária e secundária em relação a fatores desencadeantes do pé diabético. Acta Paulista de Enfermagem, São Paulo, v. 19, n. 3, p. 296-303, 2006.

OLIVEIRA, P. P. de et al. Análise comparativa do risco de quedas entre pacientes com e sem diabetes mellitus tipo 2. Revista da Associação Medica Brasileira, São Paulo, v. 58, n. 2, p. 234239, mar,/abr. 2012.

OTERO, L. M.; ZANETTI, M. L. Z.; TEIXEIRA, C. R. de S. Características sociodemográficas e clínicas de portadores de diabetes em um serviço de atenção básica à saúde. Revista Latino Americana de Enfermagem, Ribeirão Preto, n. 15, n.spe, p. 768-773, set./out. 2007.

PANAROTTO, D.; TELES, A. R.; SCHUMACHER, M. V. Fatores associados ao controle glicêmico em pacientes com diabetes tipo 2. Revista da Associação Medica Brasileira, São Paulo, v. 54, n. 4, p. 314-321, 2008.

SANTOS, C. R. B. et al. Índice de Alimentação Saudável: avaliação do consumo alimentar de diabéticos tipo 2. Nutrire: Revista da Sociedade Brasileira Alimentação e Nutrição, São Paulo, v. 34, n. 1, p. 115-129, 2009.

SANTOS, F. S.; OLIVEIRA, K. R; COLETE, D. F. Adesão ao tratamento medicamentoso pelos portadores de Diabetes Mellitus atendidos em uma Unidade Básica de Saúde no município de Ijuí/RS: um estudo exploratório. Revista de Ciências Farmacêuticas Básica e Aplicada, Araraquara, v. 31, n. 3, p. 223-227, 2010.

SOCIEDADE BRASILEIRA DE ENDOCRINOLOGIA E METABOLOGIA; ASSOCIAÇÃO BRASILEIRA DE NUTROLOGIA. Diabetes Mellitus Tipo 2: Insulinização. Projeto Diretrizes. Rio de Janeiro: Associação Médica Brasileira e Conselho Federal de Medicina, 2011.

TORRES, H. C.; PACE, A. E.; STRADIOTO, M. A. Análise sociodemográfica e clínica de indivíduos com diabetes tipo 2 e sua relação com o autocuidado. Cogitare Enfermagem, v. 15, n. 1, p. 48-54. 2010. 
WHO. Global recommendations on physical activity for health. Geneve: World Health Organization; 2010.

Recebido em: 21 fev. 2013. Aprovado em: 29 abr. 2013. 